\title{
Dendritic Pattern Formation
}

\author{
Y. Saito*, G. Goldbeck-Wood and H. Müller-Krumbhaar \\ Institut für Festkörperforschung der KFA Jülich, Postfach 1913, D-5170 Jülich, FRG
}

Received May 25, 1978; accepted June 2, 1987

\begin{abstract}
The formation of dendritic crystals in two dimensions by crystallization from a supersaturated solution is studied by a Green's function method. The dendrites develop sidebranches in qualitative agreement with experiments in three dimensions. The size and the growth rate of the primary dendrite scales with respect to supersaturation and crystalline anisotropy in agreement with the results for needle-crystals. The wavelength of the sidebranches scales with the stability length as conjectured.
\end{abstract}

\section{Introduction}

The growth of dendritic crystals like snowflakes occurs frequently, when the relevant transport of heat or matter takes place in the fluid medium ahead of the moving interface. To be precise, the fluid must be supercooled or supersaturated, thereby being in a metastable state against the formation of the crystalline phase [1].

The introduction of surface tension with crystalline anisotropy was recently found [2-8] to select a needle-shaped almost parabolic form from the continuous set of Ivantsov parabolas, while in previous approximate treatments [1] of the surface tension the continuous set seemed to survive. In fact, surface tension causes an essential singularity turning the mathematical problem into a nonlinear eigenvalue problem. The result is a discrete set of needle solutions [2-7]. A recent stability analysis [5] of the needle-solutions indicates that the fastest moving solution is stable in the moving frame of reference and thus the natural candidate for the true dendrite. Experiments [9-12] on the other hand essentially always show dendrites, i.e., needles with sidebranches.

The presently available results on the existence of a needle crystal solution are usually based on the symmetric model $[3,4,7]$, where diffusion of heat takes place symmetrically both in the solid and in the melt. Experiments in two dimensions [9] are usually based on the one-side diffusion of material within the liquid rather than on diffusion of heat. A recent analysis [6] of the one-sided model shows the relation between the needle solution for the crystallisation and the Saffmann-Taylor problem, but is not yet as detailed as the results on the symmetrical model. The results on the boundary-layer model (BLM) [8] are not conclusive in the experimentally interesting range of small deviation from equilibrium although they seem to reproduce many details of the full problem. Despite some remarkable progress up to now it is still an open problem, how to describe the nonlinear mode of operation of a sidebranch-producing dendrite, but some progress has been made recently. The best experimental results for free dendritic growth were obtained in three dimensions $[11,12]$, in two dimensions there are problems with the non-planarity of the interface normal to the side-

\footnotetext{
* Permanent address: Physics Dept., KEIO-University, Yokohama, Japan.
}

walls [9]. A further problem is the quantitative control of anisotropy both in two and three dimensions.

We have performed a numerical simulation of the full diffusional problem in two dimensions on the one-sided model [13]. This is relevant for comparison with experiments on dendritic growth from solution. The well-known model [1-6] is defined as follows.

$$
\begin{aligned}
& \frac{\partial}{\partial t} u(x, z, t) \equiv 0=\nabla^{2} u+\frac{v}{D} \frac{\partial}{\partial z} u \\
& u_{\mathrm{s}}=\Delta-d_{0}\left(1-\varepsilon_{\mathrm{m}} \cos (m \theta)\right) K \\
& -D \hat{n} \nabla u_{\mathrm{s}} \approx v \hat{n}_{\mathrm{z}}
\end{aligned}
$$

Expression (1) is the diffusion equation in quasistationary approximation in a frame of reference moving at velocity $v$ in $z$ direction. $\mathrm{Eq}(2)$ is the boundary condition for the diffusion field $u$ at the interface, at infinity one has $u=0$. Equation (3) is the conservation law for the solute or impurity at the interface. $u(x, z, t)$ is the normalized diffusion field [1], $\Delta=0 \ldots 1$ is the normalized supercooling, $D$ the diffusion coefficient, $d_{0}$ the capillary length, $\varepsilon_{\mathrm{m}}$ the strength of the $m$-fold crystalline anisotropy and $K$ the curvature of the interface. For $d_{0}=0$ one obtains Ivantsov-parabolas with tip-radius $R_{0}=p l$, where $l=2 D / v$ is the diffusion length, and the Peclet number $p$ is obtained from

$\Delta=\sqrt{\pi p} \exp (p) \operatorname{erfc}(\sqrt{p}) ; 2$-dim.

$\Delta=p \exp (p) E_{1}(p) ;$ 3-dim

(The three-dimensional relation (4b) is included here just for comparison).

In principle the anisotropy also enters in eq. (3), but in the limit of small $\Delta$ (or for a segregation-coefficient $\approx 1$ ) we may ignore it for better comparison with existing results on the two-sided thermal model. Equation (1) then is defined on the liquid side of the interface only.

The use of the quasistationary approximation here is physically meaningful [1] for parameter-values where the diffusion length is large compared to other typical lengthscales like radius of the tip of the dendrite or distance between sidebranches. At least for the smaller supercoolings used here this is definitely the case.

We have converted (1-3) into an integral form by using Green's formulas

$$
\begin{aligned}
\int \mathrm{d} \Omega & \left\{g L u-u L^{+} g\right\} \\
= & -\oint \mathrm{d} \Gamma\left\{g \frac{\partial u}{\partial n}-u \frac{\partial g}{\partial n}+\frac{2}{l} n_{\mathrm{z}} g u\right\}
\end{aligned}
$$

where $L$ is the differential operator defined in eq. (1) for the spatial derivations, $L^{+}$is the adjoint operator, $\boldsymbol{n}$ is the normal vector on the surface $\Gamma$ directed into the liquid region $\Omega$. The 
Green's function is defined by

$L_{r}^{r} g(\boldsymbol{r}, \boldsymbol{r})=-\delta(\boldsymbol{r}-\boldsymbol{r})$

and can be given in closed form [13].

Evaluating eq. (5) formally for a point $r$ on the surface, obtains

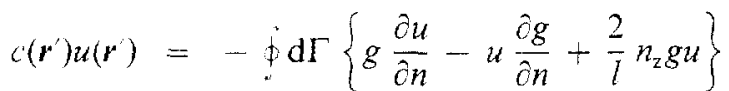

The coefficient $c(\boldsymbol{r})$ would be unity in the bulk of the liquid. If we discretize the boundary into a polygon with an inner angle $\varphi\left(\boldsymbol{r}^{\prime}\right)$ at a corner $\boldsymbol{r}^{\prime}$, then $c\left(\boldsymbol{r}^{\prime}\right)=\varphi\left(\boldsymbol{r}^{\prime}\right) / 2 \pi$. This discretization into a fine mesh along the boundary converts (eq. (7)) into an $N \times N$ matrix problem for the unknown normal derivatives of $u$, and with eq. (3) for the normal velocities. We start with a parabola pointing in $z$-direction symmetrical about the $z$-axis. $u\left(r^{\prime}\right)$ then is given at the polygonal corners by eq. (2). The Green's function depends on the yet unknown velocity $v$ of the moving frame. We start with the velocity of the corresponding Ivantsov parabola. Then we define a time step $\delta t$ and displace the interface into normal direction. We now have the new velocity from the tip of the deformed parabola and can repeat the process, simulating growth of the crystallization front.

To handle the asymptotic tail of the dendrite we have divided the interface into three parts along $z$, a tip-region, a transition-region and the tail. The tail is an Ivantsov parabola of length $>5 l$ defined by the growth rate $v$. It is displaced along with the tip. Carefull handling of this tail is important for the global conservation of the field-variable (chemical concentration or energy, resp.), as was also noticed in Ref. [7]. The missing sidebranch structure there does not seem to be important as we conclude from extrapolations to infinite system size, but it may affect the noise in the system. The tip-region is fully treated as described above. In the transition region at each time step the diffusion flux is calculated as above, but the displacement of each point is continuously modified down the curve such that it always connects to the tail. Time step and grid-spacing are dynamically adjusted, the typical grid-spacing being $<0.05 R_{0}$, smaller than used for the needle-crystal calculation [7]. Further details of the numerical procedure will be published elsewhere.

The result of such a calculation is shown in Fig. 1 as stroboscopic picture at equal time-intervals with $d_{0}=0.001$. The other parameters $\Delta=0.25, \varepsilon_{4}=0.1$ are comparable to typical experimental values, since from $\Delta$ a peclet number $<0.03$ follows, while $\varepsilon_{4}$ corresponds to the anisotropy of the capillary length of succinonitrile. The 1.h.s. in growth direction shows the tip-region only, the r.h.s. includes the transition-region, the parabolic tail is not shown. The dendrite converges to a stationary side-branch producing state independent of initial conditions. The dependence of the results on the length of the tip region considered was very weak, as long as $\approx 3$ sidebranches were contained. We have varied the length of the tip region, the grid-spacing and the initial condition for each of the parameter-sets $\Delta=(0.5$, $0.25) ; \varepsilon_{4}=(0.05,0.1,0.15)$ for an extrapolation to infinite length and zero mesh. The growth rate $v$ for the needle crystal is predicted [7] to scale as

$\sigma \equiv \frac{d_{0}}{2 D p^{2}} v=\sigma\left(\varepsilon_{4}\right)$

being a function of the anisotropy.

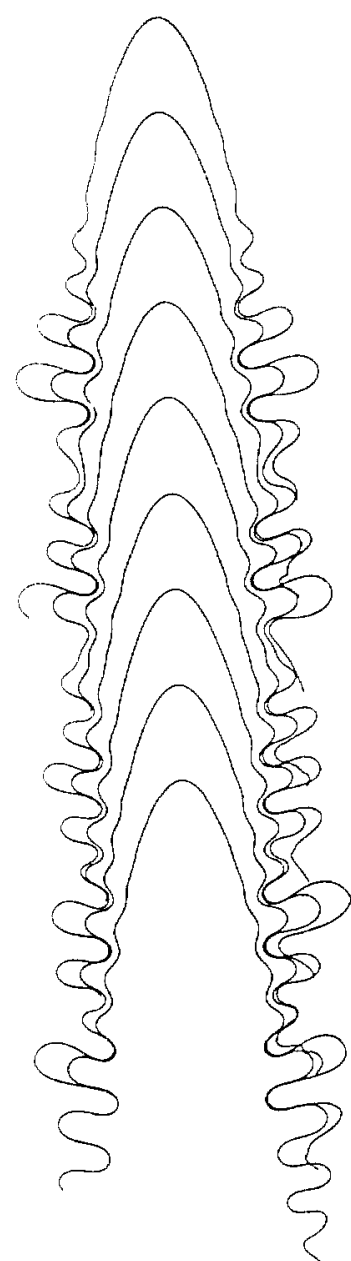

Fig. I. Stroboscopic plot of the simulation of an almost parabolic dendrite. Parameters are $\Delta=0.25, \varepsilon_{4}=0.1$. Graphs were produced at equal timeintervals.

The results for the scaled growth-rates versus anisotropy are shown in Fig. 2, compared with the needle-results [7] for the symmetrical model at $\Delta \rightarrow 0$. Dendritic growth requires nonzero anisotropy. The raw data already scale with $\varepsilon_{4}$ as predicted [7] apart from a constant factor of $\approx 2$, the extrapolated data (broken line) are only by a constant factor of $\approx 1.3$ higher than the prediction. We estimate a maximal error of 20 percent for the extrapolation. A recent calculation for needles in the one-sided model [14] gives $\sigma$-values larger by exactly a factor 2 .

Replacing $p^{2} \rightarrow \Delta^{4}$ in the definition of $\sigma$ (as suggested in the limit $p \rightarrow 0$ ), the scaled numerical data obtained at two $\Delta$-values would differ by a factor 3 ! This shows, that the results scale as $v \sim p^{2}$ at least up to $\Delta=0.5$, in agreement with a recent prediction for the needle [14].

In Fig. 3 we show the ratio of the tip actual radius $R$ and the corresponding Ivantsov radius $R_{0}$ versus anisotropy in comparison with data for the symmetrical needle [7]. Again we find striking agreement.

In Fig. 4 we show the scaled wavelength of the sidebranches plotted versus anisotropy. We have defined the inverse wavelength $\lambda^{-1}$ as the number of sidebranches produced per time unit, divided by the growth rate $v$. This appears to be a rather "robust" definition of the wavelength. Definitions based on geometrical considerations suffer from the facts that the wavelength may slightly vary in the tip-region and that sidebranches once generated may disappear or split during the nonlinear coarsening process further down the shaft. The 


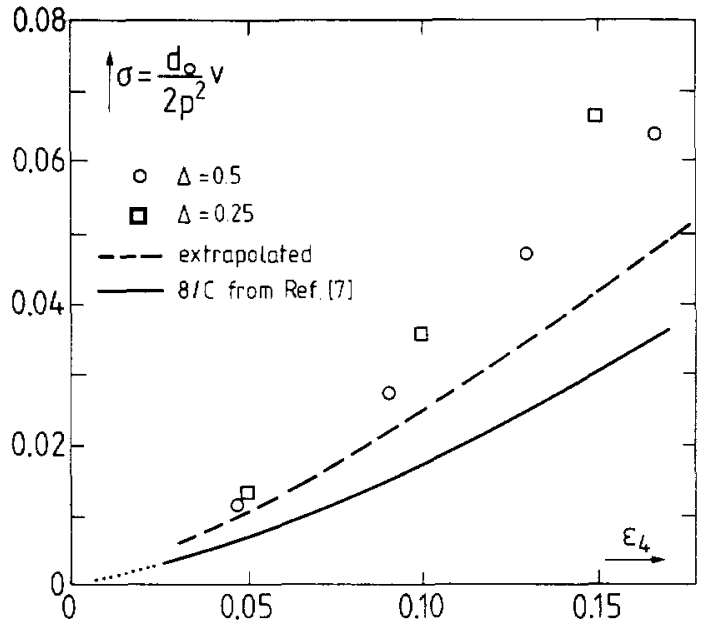

Fig. 2. Normalized growth rate versus anisotropy for $\Delta=0.25,0.5$. The broken line is our extrapolation to zero grid-spacing and infinite size, the full curve corresponds to the needle [7] in the symmetrical model. The scaling appears to be universal.

scaling relation

$\lambda \gtrsim \lambda_{\mathrm{s}}=2 \pi \sqrt{d_{0} l}$

used in Fig. 4 in dimensionless form was suggested by our previous studies [1]. Recent approximate analytical treatments $[15,16-18]$ for the evolution of a wavepacket seem to confirm this scaling with $\sqrt{\sigma}$, at least at a fixed distance from the tip.

Note that the scaling form eq. (9) of Fig. 4 contains already the dependence on supercooling $\Delta$ and anisotropy $\varepsilon_{4}$. In other words: Both the Ivantsov radius $R_{0}$ and the wavelength scale with $\lambda_{s}$. In the first case the scale factor depends on $\sigma$, in the latter case it appears to be constant. This scaling seems to contain the dependence on anisotropy, supercooling and even dimensionality.

There are, of course, a number of important points rather open. The coarsening of the sidebranches down the shaft caused by competition between them, for example, is not

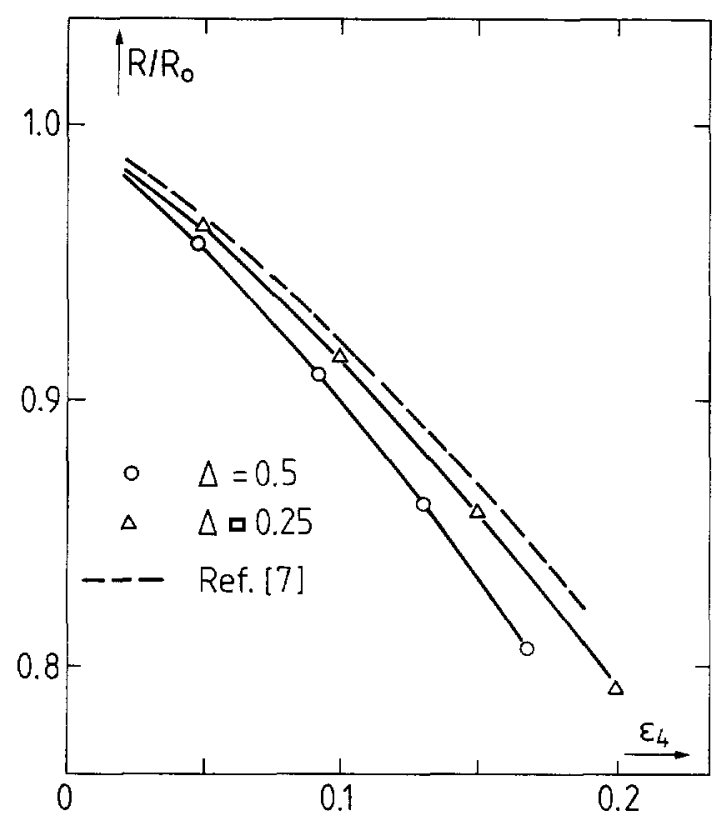

Fig. 3. Ratio of actual tip-radius $R$ over Ivantsov-radius $R_{0}$ corresponding to the same growth rate, plotted versus anisotropy. Again the scaling appears to be universal.

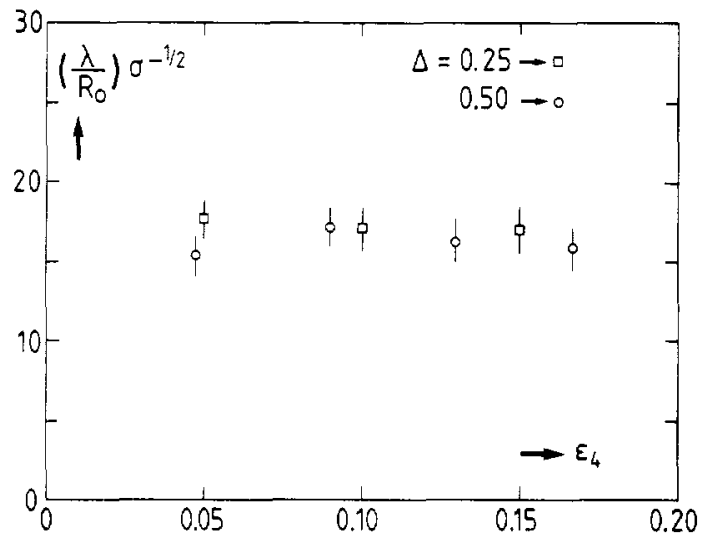

Fig. 4. Scaled wavelength $\dot{\lambda}$ of sidebranches versus anisotropy. This supports the scaling hypothesis $\lambda \sim \lambda_{\mathrm{s}}$ independent of $\Delta$ and $\varepsilon_{4}$

understood. While our computational scheme is obviously competitive with experiments concerning the questions discussed above, we do not see a simple way to extend this to the coarsening problem. A three dimensional calculation base on a mode-analysis in the present frame appears to be feasible. Kinetic anisotropy could also be included, but even less is known experimentally about this. Finally, the closely related problems of directional solidification and eutectic growth still pose a number of intriguing questions in addition to their technological relevance.

\section{References}

1. Langer, J. S., Rev. Mod. Phys. 52, I (1980); Langer, J. S., MüllerKrumbhaar, H., Acta. Metall. 26, 1681; 1689, 1697 (1978); MüllerKrumbhaar, H. and Langer, J. S., Acta. Metall. 29, 145 (1981).

2. Pelce, P. and Pomeau, Y., Studies in Appl. Math. (to appear).

3. Hong, D. C. and Langer, J. S., Phys. Rev. Lett. 56, 2032 (1986).

4. Kessler, D., Koplik, J. and Levine, H., Proceedings of the NATO A.R.W. on "Pattern, Defects and Microstructures in Non-Equilibrium Systems", Austin, Texas, March (1986); Kessler, D. and Levine, H., Phys. Rev. A. 33, 2621, 2634 (1986).

5. Kessler, D. and Levine, H., "Stability of Dendritic Crystals", Phys. Rev. Lett. (1986).

6. Kessler, D., Koplik, J. and Levine, H., "Dendritic Growth in a Channel" Phys. Rev. A (1986).

7. Ben-Amar, M. and Pomeau, Y., Europhysics Lett. 2, 307 (1986); and "Theory of the Needle Crystal" (preprint).

8. Ben-Jacob, E., Goldenfeld, N., Langer, J. S. and Schön, G., Phys. Rev. Lett. 51, 1930 (I983); Phys. Rev. 29, 330 (1984); Ben-Jacob, E., Goldenfeld, N., Kotliar, B. G. and Langer, J. S., Phys. Rev. Lett. 53, 2110 (1984).

9. Honjo, H., Ohta, S. and Sawada, Y., Phys. Rev. Lett. 55, 841 (1985).

10. Ben-Jacob, E., Godbey, R., Goldenfeld, N., Koplik, J., Levine, H., Mueller, T. and Sander, L., Phys. Rev. Lett. 55, 1315 (1985).

11. Glicksman, M. E., Schaefer, R. and Ayers, J., Metall. Trans. A 7, 1747 (1976); Huang, S. and Glicksman, M., Acta. Metall. 29, 701, 717 (1981); Glicksman, M. E. and Singh, B., in "Solidification and FluidDynamics", Proceedings of a workshop at Giesserei-Institut der RWTH-Aachen, Aachen, (1984).

12. Dougherty, A., Kaplan, P. and Gollub, J., Phys. Rev. Lett. 58, 1652 (1987).

13. Goldbeck, G., "Strukturbildung bei dendritischer Kristallisation" (unpublished); Müller-Krumbhaar, H, Goldbeck, G. and Saito, Y., in Proceedings of the OJI-Seminar on Crystal Morphology and Growth Units, Yamagata, Japan, (1985); Saito, Y., Goldbeck-Wood, G. and Müller-Krumbhaar, H., Phys. Rev. Lett. 58, 1541 (1987)

14. Misbah, C. (preprint).

15. Caroli, B., Caroli, C., Misbah, C. and Roulet, B., (preprint).

16. Pieters, R. and Langer, J. S., Phys. Rev. Lett. 56, 1948 (1986).

17. Caroli, B., Caroli, C. and Roulet, B., (preprint).

18. Barber, M. N., Barbieri, A. and Langer, J. S., (preprint). 